Rev. Bras. Saúde Prod. Anim., Salvador, v.16, n.2, p.377-386 abr./jun.., 2015 http://www.rbspa.ufba.br

\title{
Curvas de crescimento de cordeiros da raça Ile de France criados em confinamento
}

\author{
Growth curves for Ile de France ram lambs raised in feedlot
}

\author{
FALCÃO, Paula Fernanda ${ }^{1}$; PEDROSA, Victor Breno ${ }^{1 *}$; MOREIRA, Raphael Patrick ${ }^{1}$; \\ SIEKLICKI, Michelli de Fátima ${ }^{1}$; ROCHA, Caroline Gomes ${ }^{1}$; SANTOS, Izaltino \\ Cordeiro $^{1}$; FERREIRA, Evandro Maia ${ }^{1}$; MARTINS, Adriana de Souza ${ }^{1}$
}

\footnotetext{
${ }^{1}$ Universidade Estadual de Ponta Grossa, Departamento de Zootecnia, Ponta Grossa, Paraná, Brasil.

*Endereço para correspondência: vbpedrosa@uepg.br
}

\section{RESUMO}

O objetivo com este trabalho foi avaliar os modelos não lineares que melhor descrevem a curva de crescimento de ovinos machos da raça Ile de France criados em confinamento no estado do Paraná. Para tanto, foram realizadas pesagens quinzenais no período de fevereiro de 2013 a junho de 2013, desde o nascimento até o abate (120 dias) de 31 machos da raça Ile de France. As curvas de crescimento foram obtidas pelos modelos não lineares de Brody, Von Bertalanffy, Gompertz e Logístico em que foram considerados os parâmetros da taxa de maturação $(\mathrm{K})$, peso assintótico $(\mathrm{A})$, idade do animal $(\mathrm{t})$, constante de integração que determina a proporção de crescimento após nascimento (B) e proporção do valor assintótico em que ocorre o ponto de inflexão da curva $(\mathrm{m})$, de acordo com o modelo adotado. Os parâmetros foram estimados pelo método Marquardt aplicado ao procedimento NLIN do programa estatístico SAS e os critérios utilizados para verificar a precisão do ajuste das curvas foram o quadrado médio do resíduo e o coeficiente de determinação. Os modelos Brody, Von Bertalanffy, Gompertz e Logístico resultaram respectivamente em 26,$4579 ; 26,1668 ; 26,1360$ e 26,1582 para o quadrado médio do resíduo e 0,$8130 ; 0,9580$; 0,9587 e 0,9580 para o coeficiente de determinação. Considerando os critérios adotados, o modelo Gompertz, seguidopor Logístico e Von Bertalanffy, apresentaram o melhor ajuste para descrever o crescimento dos machos da raça Ile de France.

Palavras-chave: desenvolvimento ponderal, Gompertz, precocidade

\section{SUMMARY}

The objective of this study was to evaluate the nonlinear models that best describe the growth curve of ram lambs Ile de France created in intensive system in the state of Paraná. Therefore, there were weighed fortnightly during the period from February 2013 to June 2013, from birth to slaughter (120 days) of 31 Ile de France ram lambs. Growth curves were obtained by nonlinear models Brody, Von Bertalanffy, Gompertz and Logistic in which parameters were considered maturation rate $(\mathrm{K})$, asymptotic weight $(\mathrm{A})$, age of the animal $(\mathrm{t})$ is given by integration determining the proportion of growth after birth (B) and the proportion of asymptotic value at which the inflection point occurs curve $(\mathrm{m})$ in accordance with the adopted model. The parameters were estimated by Marquardt applied to the NLIN procedure of SAS statistical program and the criteria used to verify the accuracy of the fit of the curves were the mean square of the coefficient of determination. The Brody, Von Bertalanffy, Gompertz and Logistic resulted respectively in $26.4579 ; 26.1668 ; 26.136 ; 26.1582$ for the mean square and $0.813 ; 0.958 ; 0.958 ; 0.958$ for the coefficient of determination. Considering the criteria assumed the Gompertz model, followed by Logistic and Von Bertalanffy, presented the best fit to describe the growth of Ile de France ram lambs.

Keywords: Gompertz, precocity, weight development 
Rev. Bras. Saúde Prod. Anim., Salvador, v.16, n.2, p.377-386 abr./jun.., 2015 http://www.rbspa.ufba.br

\section{INTRODUÇÃO}

A ovinocultura de corte é uma das atividades com maior potencial de crescimento dentre as atividades pecuárias no Brasil (BARRETO NETO, 2010; GERON et al., 2012). Parte desta potencialidade está relacionada ao aumento do consumo da carne, estimulado pelo abate de animais precoces que proporcionam carne mais macia e com menor teor de gordura (MORENO et al., 2010). No entanto, para que esse processo seja economicamente viável é necessária a utilização de raças de rápido crescimento, permitindo a produção de cordeiros precoces (PILAR et al., 2013). A raça francesa Ile de France teve origem a partir do cruzamento das raças Merino e Leicester com o intuito de constituir um animal adaptado a produção de carne, com carcaça pesada e com bom desenvolvimento nas regiões nobres como pernil, lombo e paleta (PEREZ, 2008). Em um estudo da raça, Maia et al. (2012) destacaram que a composição genética dos animais Ile de France propicia elevadas taxas de ganhos de peso, possibilitando o abate de cordeiros mais jovens.

De acordo com Oliveira et al. (2010), uma maneira consistente de analisar a eficiência produtiva de ovinos é por meio do estudo de curvas de crescimento. Essas curvas relacionam o peso do animal, com a idade, e utilizam modelos não lineares para condensar grande volume de informação em pequeno conjunto de parâmetros que podem ser interpretados biologicamente (TEIXEIRA et al., 2011). O ajuste de funções de crescimento é uma ferramenta fundamental no planejamento da produção animal, pois permite obter informações precisas sobre ganho de peso dos animais, além de informações de prognósticos futuros para animais do mesmo grupo racial sob a mesma situação ambiental (SARMENTO et al., 2006).

A diversidade genética e variabilidade ambiental podem resultar em diferentes curvas de crescimento para uma mesma espécie de animal. Assim, existem diversos modelos de crescimento que têm a finalidade de proporcionar um método estatístico que possa indicar de forma objetiva o modelo que melhor se ajusta aos dados analisados (MALHADO et al., 2008). O ajuste dessas curvas tem sido abordado em diversos estudos com ovinos no país (LÔBO et al., 2006; MALHADO et al., 2009; SOUZA et al., 2013) no entanto, não há relatos de análise das curvas de crescimento para a raça Ile de France. Desta maneira, este trabalho teve por objetivo avaliar quais modelos não lineares, entre Brody, Von Bertalanffy, Gompertz e Logístico, melhor descrevem as curvas de crescimento de cordeiros da raça Ile de France.

\section{MATERIAL E MÉTODOS}

O experimento foi conduzido na Fazenda Escola Capão da Onça (FESCON) situada a latitude $25^{\circ} 05^{\prime} 49^{\prime \prime}$ sul e longitude $50^{\circ} 03^{\prime} 11^{\prime}$ ' oeste, pertencente à Universidade Estadual de Ponta Grossa UEPG, no município de Ponta GrossaPR. O clima da região é considerado subtropical úmido mesotérmico $(\mathrm{Cfb}) \mathrm{de}$ acordo com a classificação de Köppen, com temperatura média anual de $18^{\circ} \mathrm{C}$. A precipitação pluviométrica média anual é de 1600 à $1800 \mathrm{~mm}$ e umidade relativa de $70-75 \%$ ao ano.

A propriedade possui área de 312,11 hectares, das quais 6 hectares foram destinados à criação de ovinos de corte em sistema intensivo. Para 0 
Rev. Bras. Saúde Prod. Anim., Salvador, v.16, n.2, p.377-386 abr./jun.., 2015 http://www.rbspa.ufba.br ISSN 15199940

experimento foram utilizados 31 machos da raça Ile de France, confinados desde o nascimento até o abate, aos 120 dias de idade, submetidos a uma oferta de concentrado 4 vezes ao dia, com média de fornecimento equivalente a $3 \%$ do peso corporal. $\mathrm{O}$ alimento concentrado foi composto por milho triturado, farelo de soja, farelo de trigo, sal comum, cloreto de amônia e suplemento mineral vitamínico. Além disso, foi disponibilizada diariamente silagem de milho em quantidade equivalente a $1,5 \mathrm{~kg} / \mathrm{animal} / \mathrm{dia}$.

$\mathrm{O}$ peso corporal dos cordeiros foi determinado por meio de pesagem quinzenal no período de fevereiro de 2013 a junho de 2013, utilizando uma balança móvel mecânica (marca: Açores; modelo: 602 SM) com capacidade de $300 \mathrm{~kg}$. Os pesos considerados foram o peso ao nascimento (PN), aos 15 (P15), aos 30 (P30), aos 45 (P45), aos 60 (P60), aos 75 (P75), aos 90 (P90), aos 105 (P105) e aos 120 dias de idade (P120), sendo este último o peso ao abate. As pesagens foram realizadas no período da manhã após período de jejum de sólidos de doze horas.

Para análise estatística, foram ajustadas as curvas de crescimento de modelos não lineares de Brody, Von Bertalanffy, Gompertz e Logístico (Tabela 1). Os parâmetros utilizados nas curvas de crescimento representadas pelas letras (Y) peso corporal ao abate, (A) peso assintótico indicando um peso médio do animal livre de variações sazonais, $(\mathrm{K})$ taxa de crescimento referente à maturidade indicando a velocidade de crescimento do animal, (t) idade do animal, (B) constante relacionada ao peso inicial e $(\mathrm{m})$ responsável pela forma e determinação do ponto de inflexão da curva (ponto em que o animal passa de uma fase de crescimento inibitório atingindo um ponto de crescimento maior).
Em relação aos modelos não-lineares, não é possível resolver o sistema de equações formado diretamente, como ocorre no caso linear, uma vez que a resolução do sistema é dependente dos próprios parâmetros a serem estimados. Dessa forma, usa-se um processo iterativo para obtenção das estimativas dos parâmetros, o qual começa com valores iniciais, atribuídos aos próprios parâmetros a serem estimados. Calculase, então, a soma de quadrado do erro e, a cada passo, obtém-se um conjunto de estimativas atualizadas até $o$ procedimento convergir para um vetor final de estimativas, obtendo-se a soma mínima de quadrados do erro (SARMENTO et al., 2006).

Tabela 1. Forma geral dos modelos não lineares

\begin{tabular}{lc}
\hline Modelos & Forma geral \\
\hline Bertalanffy & $y=A\left(1-B e^{-k t}\right)^{3}+\varepsilon$ \\
Brody & $y=A\left(1-B e^{-k t}\right)+\varepsilon$ \\
Gompertz & $y=A e^{B e(-k t)}+\varepsilon$ \\
Logístico & $y=A\left(1+e^{-k t}\right)^{-\mathrm{m}}+\varepsilon$ \\
\hline
\end{tabular}

Os critérios utilizados para a escolha do modelo que melhor descreveu a curva de crescimento foram: quadrado médio do resíduo (QMR) calculado por meio da divisão da soma de quadrados do resíduo pelo número de observações, que é o estimador de máxima verossimilhança da variância residual, para que se pudesse comparar o QMR dos diferentes modelos, visto que possuem números diferentes de parâmetros a serem estimados; o coeficiente de determinação $\left(\mathrm{R}^{2}\right)$ calculado como o quadrado da correlação entre os pesos observados e estimados, por meio de uma análise de regressão linear que considera o peso predito pelo modelo não linear como variável dependente e o peso observado do 
animal como variável independente (SOUZA et al., 2011).

Após a escolha do melhor modelo, foi calculada a taxa de crescimento absoluta (TCA), obtida a partir da primeira derivada do modelo ajustado selecionado, em relação ao tempo ( $\partial \mathrm{Y} /$ dt) - (MALHADO et al., 2008). A TCA pode ser interpretada pelo ganho de peso obtido por unidade de tempo e, dessa forma, representa o ganho de peso médio diário, estimado ao longo da trajetória de crescimento avaliado. Os parâmetros dos modelos foram estimados pelo método Marquardt modificado por meio do procedimento NLIN do programa estatístico SAS, usando critério de convergência 10-8. As correlações de Pearson entre os parâmetros foram estimadas pelo procedimento CORR (SAS, 2009).

\section{RESULTADOS E DISCUSSÃO}

A Tabela 2 apresenta os resultados da estatística descritiva dos dados, em que os animais apresentaram peso médio ao nascer de $4,69 \mathrm{~kg}$. Esse resultado foi superior ao apresentado por Muller et al. (2006) que obtiveram valores de peso médio ao nascimento de $3,95 \mathrm{~kg}$ em animais cruzados Ile de France $\mathrm{x}$ Texel, mantidos em confinamento. Devido ao grande porte das ovelhas Ile de France, o maior peso ao nascimento não resulta em partos distócitos $\mathrm{e}$ devido a existência de correlação positiva desta medida com pesos em idades mais avançadas (ZISHIRI et al., 2014), pode ser considerado um fator positivo para animais deste padrão estrutural.

O peso médio ao desmame, que ocorreu aos 60 dias de idade, foi de $22,90 \mathrm{~kg}$, semelhante ao registrado também por Muller et al. (2006) de $17,39 \mathrm{~kg}$ em animais cruzados Ile de France, desmamados com a mesma idade. Em outro estudo, Moreno et al. (2010) avaliando animais puros da raça Ile de France em sistema de semiconfinamento, obtiveram pesos a desmama de $13,38 \mathrm{~kg}$ e $15,72 \mathrm{~kg}$, respectivamente para desmame aos 45 dias e aos 60 dias de idade. Isto demonstra que o sistema de criação adotado nessa pesquisa, resulta em maior ganho de peso até a desmama, o que está associado a maiores ganhos de peso pós-desmama e abates mais precoces.

O peso médio aos 120 dias de idade foi de $36,90 \mathrm{~kg}$, sendo superior quando comparado a outros estudos que utilizaram sistemas intensivos e semiintensivos para criação de ovinos lanados. Moreno et al. (2010) apresentaram resultados de $32,54 \mathrm{~kg}$ aos 124 dias para animais desmamados aos 45 dias de idade e de 32,80 aos 116 dias para animais desmamados aos 60 dias de idade, permanecendo aproximadamente 80 e 56 dias em confinamento respectivamente. Ao passo que cordeiros machos cruzados Suffolk x Corriedale tiveram uma média de peso aos 150 dias de idade de $31,8 \mathrm{~kg}$ e machos Suffolk x Ideal, também com 150 dias de idade, de $32,5 \mathrm{~kg}$ (CUNHA et al., 2000). Ou seja, quando comparados os resultados nas diferentes idades, é possível identificar o quão eficiente é o sistema de produção intensivo para machos da raça Ile de France, cabendo aos criadores identificar a possibilidade de implementar esse tipo de sistema e monitorar o crescimento dos machos entre o nascimento e o abate.

As estimativas dos parâmetros para cada modelo e os critérios utilizados para avaliar os modelos que melhor ajustar nas curvas de crescimentos são apresentados na Tabela 3. Os métodos não-lineares são alternativas interessantes para o criador determinar um modelo que 
Rev. Bras. Saúde Prod. Anim., Salvador, v.16, n.2, p.377-386 abr./jun.., 2015 http://www.rbspa.ufba.br ISSN 15199940

melhor represente o comportamento do crescimento e desenvolvimento dos animais do rebanho para que assim, permita predizer ou mesmo antecipar o manejo mais adequado a ser aplicado. Em um estudo de curvas de crescimento, Sarmento et al. (2006) citaram que em modelos não-lineares não é possível resolver o sistema de equações diretamente, como no caso dos sistemas lineares, uma vez que a resolução do sistema é dependente dos próprios parâmetros a serem estimados. Assim usa-se um processo interativo para obtenção das estimativas dos parâmetros, o qual começa com valores iniciais, atribuídos aos próprios parâmetros a serem estimados.

Tabela2. Estatística descritiva para machos da raça Ile de France criados em confinamento

\begin{tabular}{ccccc}
\hline \multirow{2}{*}{ Idade (dias) } & \multicolumn{4}{c}{ Peso $(\mathrm{kg})$} \\
\cline { 2 - 5 } & Mínimo & Máximo & Média & DP \\
\hline 0 & 3,50 & 6,50 & 4,69 & 1,05 \\
15 & 3,90 & 12,92 & 8,65 & 2,42 \\
30 & 5,96 & 20,87 & 13,20 & 3,70 \\
45 & 9,25 & 26,63 & 17,92 & 4,48 \\
60 & 10,23 & 32,10 & 22,90 & 5,23 \\
75 & 14,30 & 38,88 & 28,14 & 6,32 \\
90 & 18,11 & 42,45 & 30,95 & 6,66 \\
105 & 20,54 & 44,79 & 33,02 & 6,82 \\
120 & 24,86 & 46,37 & 36,90 & 6,25 \\
\hline
\end{tabular}

$\mathrm{DP}=$ desvio padrão $(\mathrm{kg})$.

Tabela3. Estimativas dos parâmetros (A), (B), (K), (m), quadrado médio do resíduo, coeficiente de determinação de acordo com os modelos estudados

\begin{tabular}{lcccc}
\hline \multirow{2}{*}{ Parâmetros } & \multicolumn{4}{c}{ Modelos } \\
\cline { 2 - 5 } & Brody & Von Bertalanffy & Gompertz & Logístico \\
\hline (A) & 82,6124 & 47,9554 & 43,7622 & 41,3267 \\
(B) & 0,9523 & 0,5483 & 2,2294 & - \\
(K) & 0,0046 & 0,0154 & 0,0207 & 0,0259 \\
(m) & - & - & - & 3,0531 \\
Quadrado médio do resíduo & 26,4579 & 26,1668 & 26,1360 & 26,1582 \\
Coeficiente de determinação & 0,8130 & 0,9580 & 0,9587 & 0,9580 \\
\hline
\end{tabular}

O parâmetro (A), que representa o peso assintótico do animal na raça Ile de France, foi maior em Brody $(82,6124)$ seguido de Bertalanffy (47,9554), Gompertz $(43,7622)$ e Logístico (41,3267). De acordo com estes resultados, foi evidenciado que o modelo Brody superestimou o peso dos cordeiros ao abate, quando comparados aos demais modelos. Estes resultados foram maiores comparados aos estimados em ovinos da raça Santa Inês em que, o peso assintótico (A) alcançou valores de 31,$30 ; 29,80$ e 29,38 para os modelos Brody, Bertalanffy e Gompertz respectivamente em animais mantidos 
em pastagem, desmamados aos 90 dias com peso médio de $14,23 \mathrm{~kg}$ e peso ao abate de 28 a30 kg aos 390 dias de idade (TEIXEIRA et al., 2011). Já em estudos realizados com animais cruzados Santa Inês x Texel o parâmetro (A) estimado para Brody foi de 37,66; Bertalanffy 32,16; Gompertz 30,61 e Logístico 29,14 (MALHADO et al., 2008), em que esses animais alcançaram aos 120 dias de idade o peso médio de $16,21 \mathrm{~kg}$ e $31,09 \mathrm{~kg}$ apenas aos 365 dias de idade. Além disso, comparando o referido parâmetro, $\quad$ o resultado foi consideravelmente superior aos dos animais Dorper $x$ Santa Inês, apresentados por Carneiro et al. (2007) para o modelo Logístico $(28,6809)$, indicando maior tamanho assintótico para os animais Ile de France em comparação aos cruzados de mesma idade.

Para o parâmetro $(\mathrm{K})$, que representa a taxa de maturação, foi obtido valor superior em Logístico $(0,0259)$ seguido de Gompertz $(0,0207)$, Bertalanffy $(0,0154)$ e Brody $(0,00456)$ enquanto que no estudo de Souza et al. (2011) este parâmetro resultou em0,006 para Brody, 0,009 para Bertalanffy, 0,011 para Gompertz e 0,013 para Logístico. E ainda, no trabalho de Carneiro et al. (2007) os cruzamentos resultaram em 0,0267 para $1 / 2$ Dorper $1 / 2$ Santa Inês, 0,0223 para $1 / 2$ Dorper $1 / 2$ Morada Nova e 0,0213 para $1 / 2$ Dorper $1 / 2$ Rabo Largo para o referido parâmetro, verificados no modelo Logístico. Por se tratar de um parâmetro que quanto maior o seu valor, mais precoces são os animais, as estimativas (k) do presente estudo demonstraram que os machos da raça Ile de France podem ser abatidos em idades mais jovens, o que abrevia o ciclo produtivo e o retorno econômico.

No parâmetro (B) que indica a proporção de crescimento assintótico do animal após o nascimento, o resultado foi maior em Gompertz (2,2294) seguido de Brody $(0,9523)$ e Bertalanffy $(0,5483)$, ressaltando que no modelo estatístico Logístico esse parâmetro não é considerado. Para o mesmo estudo mencionado anteriormente com ovinos da raça Morada Nova, os valores estimados foram menores quando verificado em Brody $(0,88)$, Bertalanffy $(0,47)$ e Gompertz $(1,84)$ (SOUZA et al., 2011). Para o parâmetro (m), que determina a proporção do valor assintótico em que ocorre o ponto de inflexão da curva, foi verificado para o modelo Logístico um valor de 3,0531. Em um estudo com a raça Morada Nova este parâmetro apresentou resultado aproximado ao do presente estudo $(2,53)$ (SOUZA et al., 2011).

Todos os modelos apresentaram satisfatórios valores de coeficiente de determinação $\left(\mathrm{R}^{2}\right)$ sendo de $95,87 \%$ para Gompertz, 95,80\% para Bertalanffy e Logístico, e Brody que apresentou o menor valor, de $81,30 \%$, mostrando que nesse parâmetro o modelo Brody foi o que pior se ajustou aos dados, pois maiores valores de $\mathrm{R}^{2}$ representam maior confiabilidade no modelo estimado. Os quadrados médios do resíduo (QMR) estimados para os modelos Brody, Von Bertalanffy, Gompertz e Logístico foram, respectivamente, 26,4579; 26,1668; 26,1360 e 26,1582. Assim como relatado para o coeficiente de determinação, Brody apresentou a pior estimativa de quadrado médio do resíduo, visto que para esta medida valores maiores indicam uma menor proximidade do modelo a uma estimativa ajustada aos dados observados no experimento. Os modelos Von Bertalanffy, Gompertz e Logístico apresentaram resultado semelhante, com pequena superioridade para o método de Gompertz. Este, por ter demonstrado maior valor de $\mathrm{R}^{2} \mathrm{e}$ menor QMR, indicou melhor ajuste para as curvas de crescimento de cordeiros da raça Ile de France, seguido dos modelos Logístico e 
Von Bertalanffy. Com resultado análogo, Sarmento et al. (2006) em estudo com a raça Santa Inês, concluíram que o modelo Gompertz apresentou ajuste superior e, portanto, deve ser preferido aos demais modelos de crescimento para descrição da curva média.

A Figura 1 ilustra as curvas de crescimento ajustadas pelas funções Brody e Von Bertalanffy, juntamente com os pesos médios observados (Obs). Já a Figura 2, de modo semelhante, apresenta o comportamento das curvas de crescimento Gompertz e Logístico em comparação aos valores observados. Pôde-se observar, para todos os modelos, que a partir do $60^{\circ}$ dia há diminuição do crescimento, porém, com a continuidade de ganho de peso até o período de abate. Esse resultado pode ser interpretado não somente como diminuição do crescimento ósseo e muscular, mas também como resposta fisiológica à desmama, já que a curva apresenta breve ascensão após o $75^{\circ}$ dia, em resposta a adaptação do animal ao novo sistema nutricional.

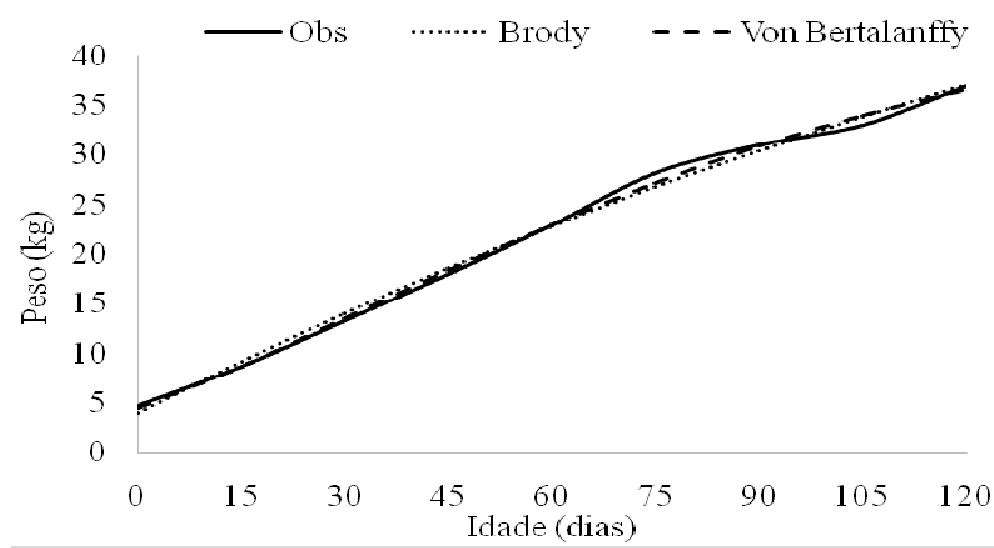

Figura1. Estimativas do peso em função da idade, obtidas pelos modelos Brody, Von Bertalanffy e observados (Obs) em cordeiros da raça Ile de France

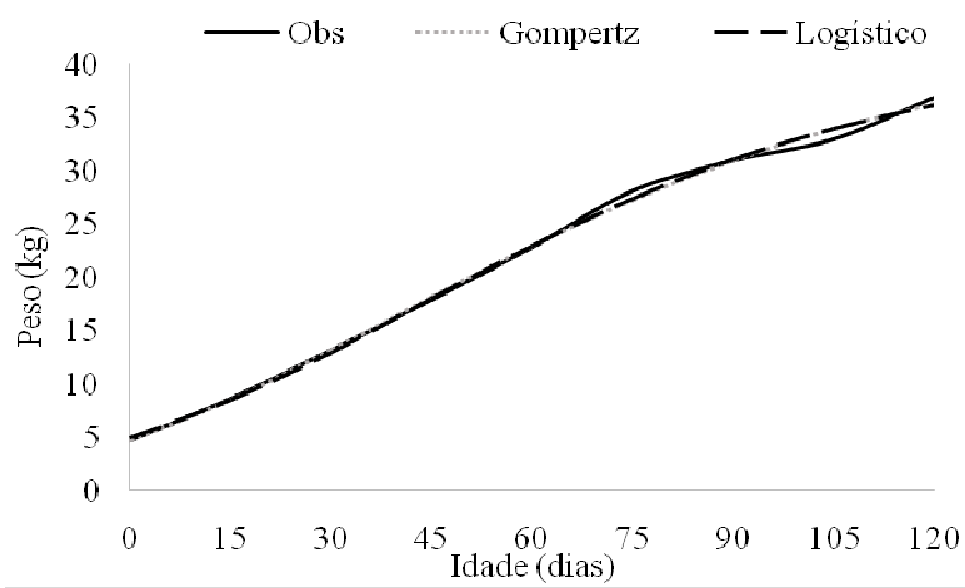

Figura 2. Estimativas do peso em função da idade, obtidas pelos modelos Gompertz, Logístico e observados (Obs) em cordeiros da raça Ile de France 
O modelo Gompertz foi selecionado para descrever a taxa de crescimento absoluto (TCA) dos cordeiros. A (TCA) foi obtida pela primeira derivada do modelo supracitado em função do tempo (Figura 3). A TCA foi crescente até atingir o valor máximo, aproximadamente $332 \mathrm{~g}$, aos 30 dias. Os animais começaram a diminuir consideravelmente o ganho de peso após os 60 dias de idade, coincidindo com o período da desmama.

Segundo Souza et al. (2011), o ponto de inflexão da curva é importante para auxiliar os produtores em programas alimentares específicos e na definição da melhor idade ao abate. Assim, estratégias nutricionais para a fase pósdesmama devem ser implementadas, de forma a minimizar os efeitos da mudança de dieta, amenizando o acentuado decréscimo da TCA após esta fase. Em ovinos criados intensivamente, esperava-se que a TCA permanecesse elevada por todo o período de confinamento, no entanto, após a desmama pôde-se observar acentuado decréscimo, demonstrando haver um limite fisiológico estrutural que restringe $\mathrm{o}$ crescimento muscular $\mathrm{e}$ ósseo.

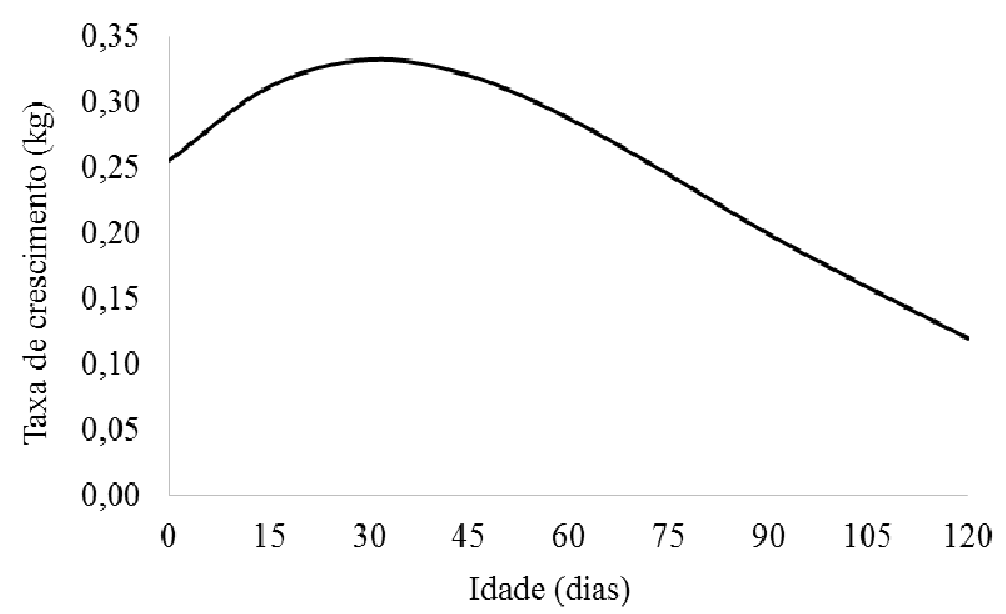

Figura 3. Taxa de crescimento absoluto estimado pela função Gompertz em cordeiros da raça Ile de France

Outro aspecto importante analisado foi a correlação entre as variáveis utilizadas nos modelos, em especial entre os parâmetros (A) e (K). Em todos os modelos utilizados os parâmetros (A) e (K) se correlacionaram negativamente (Brody $=-0,9969418$, Bertalanffy $=$ 0,9645847, Gompertz $=-0,9375803$ e Logístico $=-0,9188977$ ), demonstrando que quanto maior a velocidade de crescimento animal, menor será seu tamanho assintótico. No entanto, quanto mais precoce, mais rápido poderá ser abatido, independentemente da possibilidade de atingir peso de abate um pouco maior. Ainda, cabe ressaltar que as correlações entre os demais parâmetros não foram apresentadas por não terem resultado em efeito significativo.

As curvas de crescimento são ferramentas importantes na produção, pois permitem acompanhar $\mathrm{o}$ crescimento dos animais, a fim de se realizar os ajustes necessários ao manejo e assim, adequar o melhor peso e idade ao abate. Com base nos resultados dos parâmetros e nos critérios adotados para a escolha dos modelos mais ajustados, a curva que 
melhor descreve o crescimento de cordeiros da raça Ile de France, criados em confinamento, é a de Gompertz, seguida dos modelos Logístico e Von Bertalanffy.

\section{REFERÊNCIAS}

BARRETO NETO, A.D.

Posicionamento estratégico do setor de carnes de caprinos e ovinos no mercado de carnes brasileiro. Revista Tecnologia \& Ciência Agropecuária, v.4, n.4, p.81-85, 2010.

CARNEIRO, P.L.S; MALHADO, C.H.M; SOUZA JÚNIOR, A.A.O.S; SILVA, A.G.S; SANTOS, F.N; SANTOS, P.F; PAIVA, S.R. Desenvolvimento ponderal e diversidade fenotípica entre cruzamentos de ovinos Dorper com raças locais. Pesquisa Agropecuária Brasileira, v.42, n.7, p.991-998, 2007.

CUNHA, E.A; SANTOS, L.E.; BUENO, M.S; RODA, D.S; LEINZ, F.F; RODRIGUES, C.F.C. utilização de carneiros de raças de corte para obtenção de cordeiros precoces para abate em plantéis produtores de lã.

Revista Brasileira de Zootecnia, v.29, p.243-252, 2000.

GERON, L.J.V.; MEXIA, A.A.; GARCIA, J.; ZEOULA, L.M.; GARCIA, R.R.F.; MOURA, D.C.D. Desempenho de cordeiros em terminação suplementados com caroço de algodão (gossypium hirsutum 1.) e grão de milho moído (zea mays 1.). Archives of Veterinary Science, v.17, n.4, p.34-42, 2012.
LÔBO, R.N.B.; VILLELA, L.C.V.; LÔBO, A.M.B.O.; PASSOS, J.R.S.; OLIVEIRA, A.A. Parâmetros genéticos de características estimadas da curva de crescimento de ovinos da raça Santa Inês. Revista Brasileira de Zootecnia, v.35, p.1012-1019, 2006.

MAIA, M.D.O.; COSTA, F.D.S.; SUSIN, I.; RODRIGUES, G.H.; FERREIRA, E.M.; PIRES, A.V.; MENDES, C.Q. Effect of genotype on chemical composition and fatty acid profile of ewe lamb meat. Revista Brasileira de Zootecnia, v.41, p.986992, 2012.

MALHADO, H.M.; CARNEIRO, P.L.S.; SANTOS, P.F.; AZEVEDO, D.M.M.R.; SOUZA, J.C.; AFONSO, P.R.M. Curvas de crescimento em ovinos mestiços Santa Inês X Texel criados no sudoeste do Estado da Bahia.

Revista Brasileira de Saúde e Produção Animal [online], v.9, n.2, p.210-218, 2008.

MALHADO, C.H.M.; CARNEIRO, P.L.S.; AFFONSO, P.R.A.M.; SOUZA JUNIOR, A.A.O.; SARMENTO, J.L.R. Growth curves in Dorper sheep crossed with the local Brazilian breeds, Morada Nova, Rabo Largo, and Santa Inês.

Small Ruminant Research, v.84, p.1621, 2009.

MORENO, G.M.B.; SILVA, S.A.G.; ROSSI, R.C.; PEREZ, H.L.; LEÃO, A.G.; ZEOLA, N.M.B.L.; SOUZA, J.S.C. Desempenho e rendimentos de carcaça de cordeiros Ile de France desmamados com diferentes idades.

Revista Brasileira de Saúde e Produção Animal [online], v.11, n.4, p.1105-1116, 2010. 
MÜLLER, L.; PIRES, C.C.; TONETTO, C.J.; VOLLENHAUPT, L. da S.; MEDEIROS, S.L.P. Efeito do desmame precoce em cordeiros cruzas Ile de France $\mathrm{x}$ Texel no desempenho e nas características da carcaça. Revista

Ciência Agronômica, v.37, n.2, p.241245, 2006.

OLIVEIRA, D.C.; SOUZA, G.N.; CORDEIRO, L.L.; CARNEIRO, A.P.S.; CARNEIRO, P.L.S. Modelos de curvas de crescimento para ovinos cruzados.

Revista da Estatística UFOP, v.1, p.1-2, 2010.

\section{PEREZ, H. Desempenho Produtivo e} Reprodutivo de Ovinos Lanados. 2008. 65f. Dissertação (Mestrado) -

Universidade Estadual Paulista "Julio de Mesquita Filho”, Jaboticabal.

PILAR, R.D.C.; PERES, J.R.O.; MUNIZ, J.A.; BRESSAN, M.C. Alometria dos cortes da carcaça, em cordeiros merino australiano e cruza ile de france x merino australiano. Current Agricultural Science and Technology, v.14, n.4, p.91101, 2013.

SARMENTO, J.L.R.; REGAZZI, A.J.; SOUSA, W.H; TORRES, R.A.; BREDA, F.C.; MENEZES, G.R.O. Estudo da curva de crescimento de Ovinos Santa Inês. Revista Brasileira de Zootecnia, v.35, n.2, p.435-442, 2006.

STATISTICAL ANALYSIS SYSTEM SAS. User's guide. Version 9.2 ed. Cary: SAS Institute, USA, 2009.

SOUZA, L.A; CARNEIRO, P.L.S; MALHADO, C.H.M; PAIVA, S.R; CAIRES, D.N E BARRETO, D.L.F. Curvas de crescimento em ovinos da raça morada nova criados no estado da Bahia. Revista Brasileira de Zootecnia, v.40, n.8, p.1700-1705, 2011.
SOUZA, L.A.; CARNEIRO, P.L.S.; MALHADO, C.H.M.; SILVA, F.F.; SILVEIRA, F.G. Traditional and alternative nonlinear models for estimating the growth of Morada Nova sheep. Revista Brasileira de Zootecnia, v.42, n.9, p.651-655, 2013.

TEIXEIRA, M.C.; VILLARROEL, A.B.; PEREIRA, E.S.; OLIVEIRA, S.M.P.; ALBUQUERQUE, I.A.; MIZUBUTI, I.Y. Curvas de crescimento de cordeiros oriundos de três sistemas de produção na Região Nordeste do Brasil. Ciências Agrárias, v.33, n.5, p.2011-2018, 2011.

ZISHIRI, O.T.; CLOETE, S.W.P.; OLIVIER, J.J.; DZAMA, K. Genetic parameters for live weight traits in South African terminal sire sheep breeds. Small Ruminant Research, v.116, n.2, p.118-125, 2014.

Data de recebimento: 03/02/2014 Data de aprovação: 02/05/2015 